\title{
GOLM1-regulated EGFR/RTK recycling is a novel target for combating HCC metastasis
}

\author{
Wenwei Zhu \& Lunxiu Qin* \\ Department of General Surgery, Huashan Hospital, Cancer Metastasis Institute, Fudan University, Shanghai 200040, China
}

Received October 19,2016; accepted November 2, 2016; published online November 17, 2016

\begin{abstract}
Citation: Zhu, W., and Qin, L. (2017). GOLM1-regulated EGFR/RTK recycling is a novel target for combating HCC metastasis. Sci China Life Sci 60, 98-101. doi: $10.1007 / \mathrm{s} 11427-016-0311-\mathrm{x}$
\end{abstract}

A salient feature of metastasis is the ability of different tumor types to colonize the same or different organ sites. Many genetic and epigenetic events have been identified that contributing to the metastatic cascades, including migration, local invasion, intravasation, survival in the circulation, extravasation and colonization, and some key molecules might have dual functions promoting metastasis by providing both local advantage from malignant progression in the primary tumor and a distal advantage from infiltration of distant organs. Nevertheless, the metastatic process remains poorly understood and the insight into the molecular events that initiate and/or sustain this process remains incomplete.

The Golgi apparatus-complex is a highly dynamic organelle which is considered the "heart" of intracellular transportation. It has been demonstrated to play an active role in cell migration through posttranslational modification; and prominent changes in Golgi apparatus, with disruption at biochemical composition, structure and function levels, have been observed in human carcinogenesis and metastasis (Wlodkowic et al., 2009). The important cancer-specific hallmarks of Golgi include the aberrant glycosylation and abnormal expression of Ras GTPases, as well as dysregulation of cell migration (Wlodkowic et al., 2009). While fragmentation of the Golgi was confirmed in cancer by electron microscopy almost fifty years ago, it is only in recent years that we have begun to understand the significance of Golgi

*Corresponding author (email: qinlx@fudan.edu.cn) apparatus in cancer biology, especially in cancer metastasis and progression.

Golgi-related golgi membrane protein 1 (GOLM1, also named GOLPH2 or GP73) is a type II Golgi membrane protein with a short $\mathrm{N}$-terminal sequence in the cytoplasm, and its expression is induced by viral infection (Kladney et al., 2000). It has been characterized as a diagnostic marker for a number of cancers including hepatocellular carcinoma (HCC) (Marrero et al., 2005), however, little is known about the possible roles of GOLM1 protein in cancer metastasis. In our recent study, based on comparing the gene expression profiles of HCCs with and without extra-hepatic metastasis, we identified GOLM1 as one of the leading genes favoring HCC metastasis (Ye et al., 2016). The patients with high GOLM1 levels in HCC had a higher possibility of early tumor recurrence and reduced overall survival. Furthermore, GOLM1 depletion reduced in vitro proliferation, migration, and invasion of HCC cell lines with high levels of GOLM1, and reduced tumor growth and lung metastases in tumor xenografts in vivo. These indicate that GOLM1 promotes $\mathrm{HCC}$ growth and metastasis.

To understand how GOLM1 to regulate cancer metastasis, we further performed both biochemical and cell biological assays, and revealed that GOLM1 could physically interact with the epidermal growth factor receptor (EGFR), a member of receptor tyrosine kinases (RTKs) which integrates input from multiple signaling pathways to control cell growth, proliferation, and survival. The physical interaction between GOLM1 
and EGFR was required for the GOLM1-mediated enhancement of the downstream AKT/S6K activation and cell migration (Ye et al., 2016). Although the precise mechanistic basis for the activity of GOLM1 on RTKs signaling remains to be fully elucidated, GOLM1 represents a first-in-class Golgi oncoprotein associated with RTKs activation and cancer metastasis.

Emerging evidences support the concept that the malignant behaviors of cancer, cellular proliferation, differentiation, invasion and metastasis, are sustained by the deregulated activation of complex network of intracellular and extracellular signal transduction cascades. The growth-factor-responsive receptor tyrosine kinases have a critical role in the etiology and/or progression of human cancers through activating downstream signaling pathway. In addition to the multiple mechanisms leading to the oncogenic activation of RTKs, such as transcriptional overexpression, chromosomal translocation, point mutation, and creation of an autocrine stimulation loop, ligand-induced RTKs endocytosis/recycling has recently been demonstrated to bind and phosphorylate the downstream signaling proteins in pre-degradative intracellular compartments and to activate signaling pathways. Upon ligand binding, RTKs become catalytically active and tyrosine phosphorylated enabling the recruitment of signaling proteins to initiate downstream signaling cascades. This process is balanced by the simultaneous recruitment of endocytic proteins, which enhance RTKs internalization, allowing for their removal from the cell surface and subsequent signal termination (Sorkin and von Zastrow, 2009). Following the ligand activation, RTKs, including EGFR, are internalized through clathrin-dependent or -independent mechanisms, eventually converging to deliver cargo to early endosomes (Sorkin and von Zastrow, 2009) and finally towards one of the two fates, being routed to late endosomes/lysosomes for degradation, or being recycled back to the plasma membrane. The ensuing sorting pathways destine cargo proteins for lysosome-mediated degradation or recycling back to the plasma membrane for reactivation, thereby serving as an important means for regulating homeostasis in receptor signaling.

Given their importance in maintaining a balance in growth factor signaling, it is reasonable to expect that deregulated receptor trafficking might provide a mechanism to promote oncogenesis through delaying receptor internalization, favoring recycling over degradation. In the first case, as refer to 'internalization phase', internalized RTKs can continue to signal from endosomal compartments and it is now recognized that the ability of endosomes to serve as an intracellular signaling platform is an important component of the RTKs signaling cascade during the cancer development. More importantly, in the latter case, as refer to "recycling phase", RTKs subcellular compartmentalization and recycling have become an important theme in controlling the magnitude and specificity of the response, which is related to cell transfor- mation, proliferation, and migration. During this process, defective vasicular trafficking/recycling of RTKs enables internalized proteins to return to the plasma membrane through a recycling compartment, which is regarded as a multifaceted hallmark of malignant cells (Mosesson et al., 2008). One of the earliest known examples of an endocytotic protein with transforming activity is Huntingtin interacting protein-1, a cofactor involved with clathrin-mediated endocytosis, overexpression of which alters clathrin trafficking, leading to the delayed endosome-mediated degradation of RTKs and prolonged downstream signaling through the mitogen-activated protein kinase and phosphoinositide 3-kinase pathways (Hyun and Ross, 2004). The significance of Huntingtin interacting protein-1 upregulation is further evidenced through its correlation with poor clinical outcome in a variety of epithelial and lymphoid malignancies (Hyun and Ross, 2004). Although, the identification of bona fide oncoproteins functioning in RTKs endocytotic pathways servers for understanding the development of cancer metastasis, the mechanisms and involving molecules regulating and coordinating RTKs recycling pathway remain poorly understood.

In our study, GOLM1 is recruited to an activated EGFR/RTK cargo complex upon ligand stimulation. The formation of a GOLM1-EGFR complex is localized to a Rab11 enriched endosomal compartment, this promotes the access of EGFR into a recycling pathway, decreases the entry of EGFR into the degradative pathway, and prevents EGFR degradation. The GOLM1-dependent entry of EGFR into the recycling pathway promotes sustained downstream activation and re-localization of EGFR towards the leading edge to initiate localized signaling required for cell growth and metastasis (Figure 1).

The Golgi apparatus, especially the trans-Golgi network (TGN) is the sorting and packaging center for trafficking cargo to the endosomes, plasma membrane, preceding Golgi cisternae, and endoplasmic reticulum (ER). Recycling of RTKs to the cell surface can occur either directly from the early endosome via a "fast route", or indirectly through a "slow route", traversing the endocytic recycling compartment. In the later "slow route", the cis-Golgi receives cargo including endocytic RTKs and then progresses to the medial and trans-Golgi where it acquires various post-translational modification and finally recycled back to the cell surface or to lysosomes for degradation.

Although the RTKs transportation in different subcellular compartments is mainly modulated by Golgi apparatus, and is able to sort RTKs into recycling or degradation after ligand binding, little is known about the mechanisms of the Golgi-related molecules controlling the intracellular transportation/recycling of RTKs as well as their possible roles in cancer metastasis.

In a recent study, Christine and their c olleagues have 


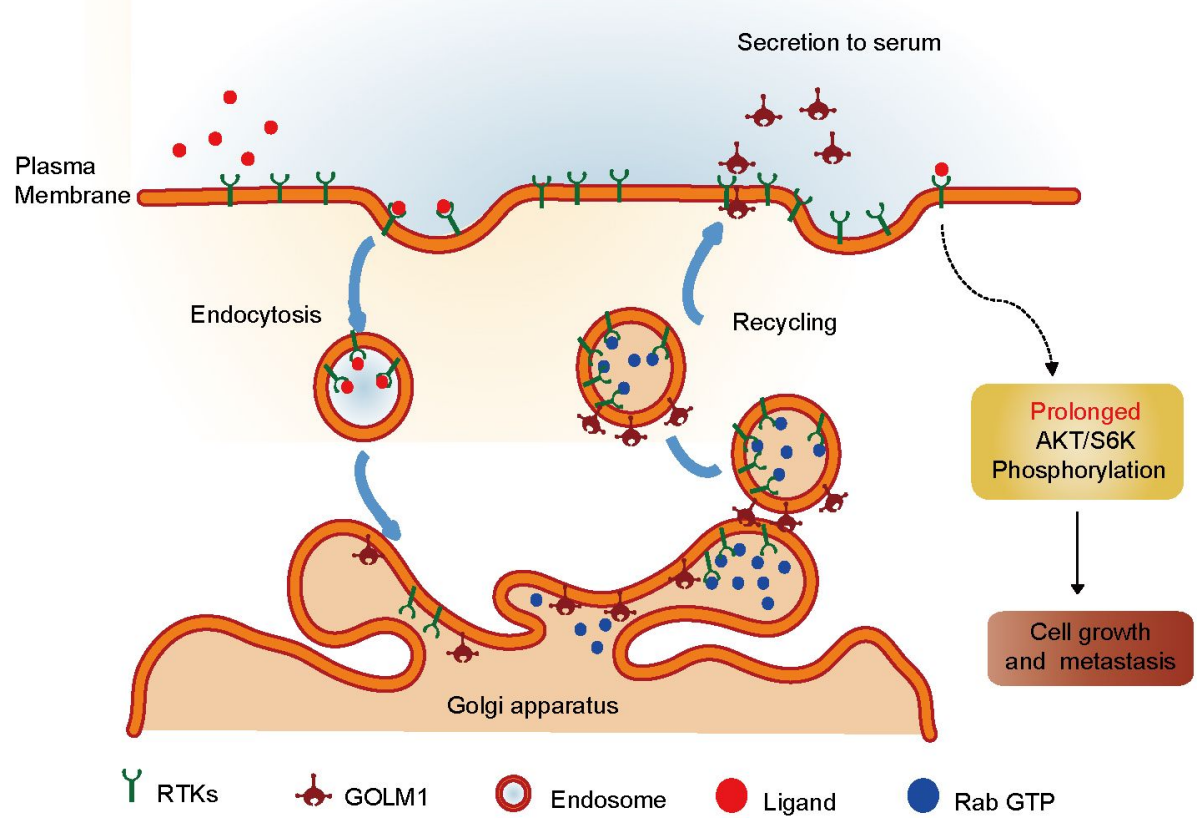

Figure 1 Illustration depicting possible routes for GOLM1-mediated RTK recycling. Following ligand binding, cell surface receptors such as RTKs are destined for internalization for receptor recycling or lysosome-mediated destruction. GOLM1 localization to the Golgi apparatus and, enhances RTKs recycling in assistance with Rab GTP to strengthen the downstream signaling cascades.

reported Golgi-localized gamma-ear containing Arf-binding protein 3 (GGA3) is recruited to an activated Met/RTK cargo complex present within the early tubular endosomal network. The formation of a GGA3-Met/RTK complex would promote the access of Met/RTK into a recycling pathway while decrease the entry of Met/RTK into the degradative pathway. GGA3-dependent entry of Met/RTK into the recycling pathway, promotes sustained ERK1/2 activation and re-localization of Met/RTK towards the leading edge to initiate localized signaling required for cell migration (Parachoniak et al., 2011). Moreover, GOLPH3, another family member of Golgi phosphoprotein, was demonstrated to localize at the peripheral structures including the endosomal compartments and the plasma membrane, which suggests that GOLPH3 may traffic to these structures, hence facilitating cargo sorting from the Golgi (Scott et al., 2009).

GOLM1, much more like GOLPH3, localizes at the cisGolgi via a lumenal targeting signal within its coiled-coil stem domain, and its retrieval from post-Golgi structures occurs via the microtubule- and PI3 kinase independent, late endosome bypass pathway, which sorts a subset of proteins, at the early endosome away from those enroute to the late endosome/lysosome for direct recycling to the Golgi apparatus (Puri et al., 2002). We have also noticed that GOLM1, similar to the structurally related protein GPP130 cycles between the Golgi and the cell surface, may be involved in RTKs intracellular trafficking and recycling following ligand binding. We speculate that GOLM1 functions as a specific cargo adaptor to assist RTKs anchoring on the TGN and recycling back to plasma membrane, rather than degradation pathway, which allows for the prolonged activation of AKT/S6K from endosomes. The biological function of endosomal GOLM1 cycling in cancer development remains to be further investigated. Future work will be required to further determine the mechanism of GOLM1-mediated RTKs intracellular trafficking and recycling, and more importantly, Golgi regulated RTKs recycling on difference cancer cells.

In summary, these findings of our study indicate that GOLM1 may promote HCC by regulation of EGFR/RTK recycling, and suggest that therapeutic targeting of GOLM1 may be a potential strategy for combating HCC metastasis. The components of these pathways might represent novel therapeutic targets against cancer; therefore, additional insight into the biological activity of GOLM1 may ultimately serve as an entry point to translate the modifiers of these pathways into clinical endpoints.

Compliance and ethics The author(s) declare that they have no conflict of interest.

Acknowledgements This work was supported by the National Natural Science Foundation of China (81472677).

Hyun, T.S., and Ross, T.S. (2004). HIP1: trafficking roles and regulation of tumorigenesis. Trends Mol Med 10, 194-199.

Kladney, R.D., Bulla, G.A., Guo, L., Mason, A.L., Tollefson, A.E., Simon, D.J., Koutoubi, Z., and Fimmel, C.J. (2000). GP73, a novel Golgi-localized protein upregulated by viral infection. Gene 249, 53-65.

Marrero, J.A., Romano, P.R., Nikolaeva, O., Steel, L., Mehta, A., Fimmel, C.J., Comunale, M.A., D'Amelio, A., Lok, A.S., and Block, T.M. (2005). GP73, a resident Golgi glycoprotein, is a novel serum marker for hepatocellular carcinoma. J Hepatol 43, 1007-1012. 
Mosesson, Y., Mills, G.B., and Yarden, Y. (2008). Derailed endocytosis: an emerging feature of cancer. Nat Rev Cancer 8, 835-850.

Parachoniak, C.A., Luo, Y., Abella, J.V., Keen, J.H., and Park, M. (2011). GGA3 functions as a switch to promote met receptor recycling, essential for sustained ERK and cell migration. Dev Cell 20, 751-763.

Puri, S., Bachert, C., Fimmel, C.J., and Linstedt, A.D. (2002). Cycling of early Golgi proteins via the cell surface and endosomes upon lumenal pH disruption. Traffic 3, 641-653.

Scott, K.L., Kabbarah, O., Liang, M.C., Ivanova, E., Anagnostou, V., Wu, J., Dhakal, S., Wu, M., Chen, S., Feinberg, T., Huang, J., Saci, A., Widlund, H.R., Fisher, D.E., Xiao, Y., Rimm, D.L., Protopopov, A., Wong, K.K., and Chin, L. (2009). GOLPH3 modulates mTOR signalling and rapam- ycin sensitivity in cancer. Nature 459, 1085-1090.

Sorkin, A., and von Zastrow, M. (2009). Endocytosis and signalling: intertwining molecular networks. Nat Rev Mol Cell Biol 10, 609-622.

Wlodkowic, D., Skommer, J., McGuinness, D., Hillier, C., and Darzynkiewicz, Z. (2009). ER-Golgi network-A future target for anti-cancer therapy. Leukemia Res 33, 1440-1447.

Ye, Q.H., Zhu, W.W., Zhang, J.B., Qin, Y., Lu, M., Lin, G.L., Guo, L., Zhang, B., Lin, Z.H., Roessler, S., Forgues, M., Jia, H.L., Lu, L., Zhang, X.F., Lian, B.F., Xie, L., Dong, Q.Z., Tang, Z.Y., Wang, X.W., and Qin, L.X. (2016). GOLM1 modulates EGFR/RTK cell-surface recycling to drive hepatocellular carcinoma metastasis. Cancer Cell 30, 444-458. 